\title{
Transition-edge superconducting antenna-coupled bolometer
}

Cynthia L. Hunt, James J. Bock, Peter K. Day, Alexey Goldin, Andrew E. Lange, et al.

Cynthia L. Hunt, James J. Bock, Peter K. Day, Alexey Goldin, Andrew E. Lange, Henry G. LeDuc, Anastasios Vayonakis, Jonas Zmuidzinas, "Transition-edge superconducting antenna-coupled bolometer," Proc. SPIE 4855, Millimeter and Submillimeter Detectors for Astronomy, (17 February 2003); doi: 10.1117/12.459110

Event: Astronomical Telescopes and Instrumentation, 2002, Waikoloa, Hawai'i, United States 


\title{
Transition-edge superconducting antenna-coupled bolometer
}

\author{
Cynthia L. Hunt ${ }^{1 \mathrm{a}}$, James J. Bock ${ }^{\mathrm{b}}$, Peter K. Day ${ }^{\mathrm{b}}$, Alexey Goldin ${ }^{\mathrm{b}}$, Andrew E. Lange ${ }^{\mathrm{a}}$, \\ Henry G. LeDuc ${ }^{\text {b }}$ Anastasios Vayonakis ${ }^{a}$, Jonas Zmuidzinas ${ }^{\text {a }}$ \\ ${ }^{a}$ California Institute of Technology, Pasadena, CA, USA \\ ${ }^{\mathrm{b}}$ Jet Propulsion Laboratory, Pasadena, CA, USA
}

\begin{abstract}
We report test results for a single pixel antenna-coupled bolometric detector. Our device consists of a dual slot microstrip antenna coupled to an $\mathrm{Al} / \mathrm{Ti} / \mathrm{Au}$ voltage-biased transition edge superconducting bolometer (TES). The coupling architecture involves propagating the signal along superconducting microstrip lines and terminating the lines at a normal metal resistor colocated with a TES on a thermally isolated island. The device, which is inherently polarization sensitive, is optimized for $140 \mathrm{GHz}$ band measurements. In the thermal bandwidth of the TES, we measure a noise equivalent power of $2.0 \times 10^{-17} \mathrm{~W} / \sqrt{\mathrm{Hz}}$ in dark tests that agrees with calculated NEP including only contributions from thermal, Johnson and amplifier noise. We do not measure any excess noise at frequencies between 1 and $200 \mathrm{~Hz}$. We measure a thermal conductance $\mathrm{G} \sim 5.5 \times 10^{-11} \mathrm{~W} / \mathrm{K}$. We measure a thermal time constant as low as $437 \mu \mathrm{s}$ at $3 \mu \mathrm{V}$ bias when stimulating the TES directly using an LED.
\end{abstract}

\section{INTRODUCTION}

The temperature anisotropy of the cosmic microwave background (CMB) is now being probed to unprecedented accuracy and sky coverage by MAP, and will be definitively mapped by Planck after its launch in 2007 . However, the polarization of the $\mathrm{CMB}$ will not be mapped sufficiently. In particular the curl-polarization, which may be used to probe the energy scale of the inflationary epoch, requires a large advance in the format of millimeter-wave bolometer arrays. SAMBA (Superconducting Antenna-coupled Multi-frequency Bolometric Array) is being developed to address these needs for the next generation of sub-millimeter astronomical detectors. SAMBA consists of a focal plane populated with microstrip antennae, whose signals are coherently added and sent to transition edge superconducting bolometers (TES) via microstrip lines. SAMBA eliminates the need for the feedhorns and IR filters normally currently used on current CMB observational instruments, such as Planck and Boomerang. The SAMBA architecture allows for a high density of pixels in the focal plane with minimal sub-Kelvin mass. As a precursor to a full monolithic high-density antenna array, we are developing a single band antenna-coupled bolometric detector.

\section{ARCHITECTURE}

The single element device presented in this paper consists of a dual slot microstrip antenna coupled to a TES (Figure 1). The electric field from the microstrip antennae propagates along planar microstrip lines, which consist of a niobium superconducting lead separated from the ground plane by $2000 \AA$ of $\mathrm{SiO}$ dielectric. The electric fields from each antenna are passively added and terminated at a thin film Au resistor located on a thermally isolated silicon nitride island. This resistor, which is designed to be used as the microstrip termination, can also be used as a heater to simulate optical

\footnotetext{
${ }^{1}$ Corresponding Author: Cynthia L. Hunt, 1200 E. California Blvd., MC 59-33, Pasadena, CA 91125. email: cynthiah@its.caltech.edu, phone(626)395-2016
} 


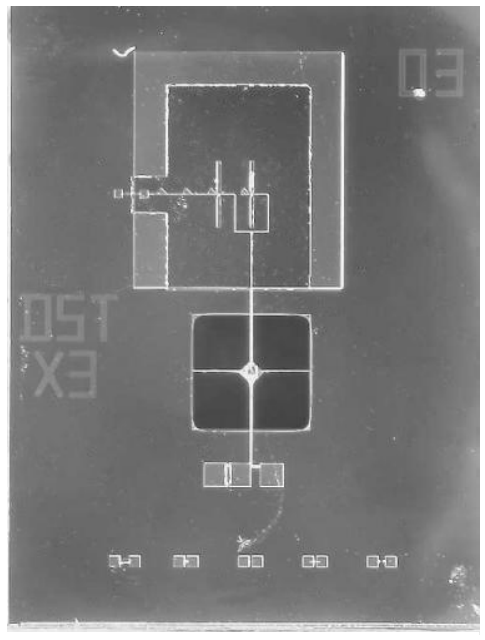

Figure 1: A fully fabricated single element device studied in this paper. This device is optimized for $140 \mathrm{GHz}$ radiation.

power. On the same island, an $\mathrm{Al} / \mathrm{Ti} / \mathrm{Au}$ TES film responds to power dissipated in the resistor, and the signal is read out with a low impedance superconducting quantum interference device (SQUID) current amplifier. We use a standard circuit for the operation and readout of a TES ${ }^{1}$.

There are several advantages of this design for a millimeter wave bolometer. One advantage is that the microstrip line can be quite long with small losses. Vayonakis et al. report $1 \%$ loss per wavelength in the dielectric at temperatures below $1.5 \mathrm{~K}^{2}$, and specifically measure this loss at $150 \mathrm{GHz}$. For a densely packed focal plane of microstrip antennas, this small loss allows the TES bolometers to be placed out of the optically exposed focal plane. Another advantage in using microstrip technology is that microstrip filters can be inserted between the antennae and the bolometer to define several bands from one pixel ${ }^{3}$. This filtering replaces conventional optical filter stacks that are massive and cumbersome in current millimeter wave experiments like Boomerang and Planck. Finally, the slot antenna design is intrinsically polarization sensitive which is ideal for making observations of the CMB polarization.

\section{RESULTS}

Bias curves at varying bath temperatures were taken to determine the relationship between power and temperature. From this, we determine a thermal conductance $\mathrm{G}=55 \mathrm{pW} / \mathrm{K}$. Devices that have not been etched and released show a transition of $414 \mathrm{mK}$, while released devices tend to transition around $360 \mathrm{mK}$. The change in the transition temperature may be a result of changes to the film upon final processing, or due to stray optical power in the dewar which would result in a lower apparent transition temperature.

To measure the time constant, we used a LED coupled to the TES via optical fiber to heat the TES island directly. This technique has the advantages of being electrically disconnected from the device and relatively quick and easy measurement to make. Although the LED does not emit light in the bandwidth of our antenna, it does simulate optical power by dissipating thermal power directly on the TES island. The LED was driven with a pulse generator and the TES response was measured on an oscilloscope. The fastest time constant measured was $437 \mu$ s biased at $3 \mu \mathrm{V}$, as shown in Figure 2. This is the effective time constant reduced by electrothermal feedback in the voltage biased TES ${ }^{4}$. Since we do not know the coupling efficiency of the LED to the island, we are not able to measure how much power is deposited into our TES.

The Au terminating resistor was used as a heater to apply a known power to the TES to study its current responsivity and linearity. Applying a bias across the resistor on the thermally isolated island deposits heat into the TES, simulating optical power in a controlled manner. While applying bias to the heater, we measure load curves, which yield current responsivity and the transient power deposited in the TES. The loop gain of the TES is given by $\mathscr{L}=-\delta \mathrm{P}_{\mathrm{b}} / \delta \mathrm{P}_{\mathrm{TOT}}, w_{h} \mathrm{~T}$ $\mathrm{P}_{\text {TOт }}$ is the total power dissipated in the device, i.e. bias power plus heater power. As the loop gain gets very large, the slope of bias power vs. heater power approaches 1. Our device shows this linear behavior to better than $1 \%$ (Figure 3 ). We also measure the current response of the TES for a given bias voltage at different applied heater powers. The current responsivity, $\mathrm{S}_{\mathrm{i}}=\delta \mathrm{I} / \delta \mathrm{P}$. Again we take the limit as the loop gain gets large and taking a DC measurement, $\mathrm{S}_{\mathrm{i}}=-1 / \mathrm{V}_{\mathrm{b}}$, which is shown in Figure 4, at a bias of $1 \mu \mathrm{V}$. In the future, we will be able to measure and compare the time constants using the on-island heater and LED technique described above.

We have measured the current noise of this device for a wide range of frequencies. Figure 5 shows the noise current of the device operated at a bath temperature of $282 \mathrm{mK}$, a bias voltage of $0.38 \mu \mathrm{V}$, and a measured effective time constant of $1.1 \mathrm{~ms}$, measured with the LED technique. Within the thermal bandwidth of the TES the noise equivalent power is $\sim 2.0 \times$ $10^{-17} \mathrm{~W} / \sqrt{ } \mathrm{Hz}$ which is in good agreement with noise theory given by ${ }^{5}$ : 


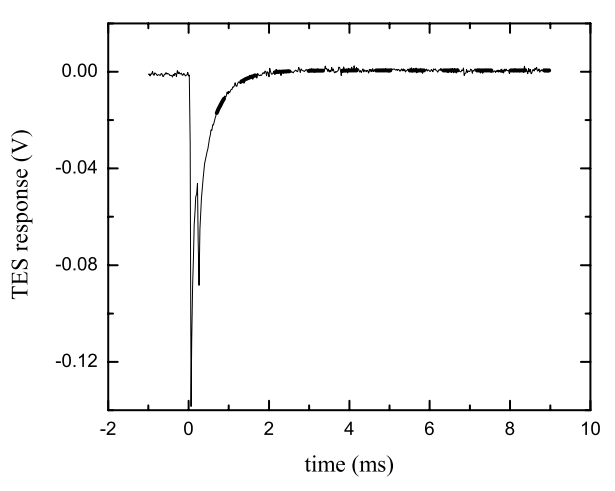

Figure 2: The fastest response of TES to a pulse from an LED coupled directly via optical fiber. Data taken at $282 \mathrm{mK}$ and $3 \mu \mathrm{V}$ bias. The exponential fit to the response is shown as the bold dashed line and indicates and effective time constant of $437 \mu$ s.

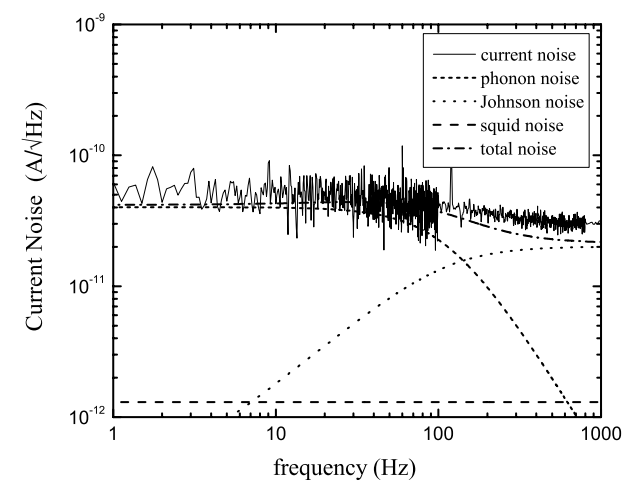

Figure 3: Measured current noise from the single element TES, biased at $0.38 \mu \mathrm{V}$. Spectra taken at different frequency bands were combined for better coverage of the range of frequencies. There is slight excess noise at frequencies above $\sim 200$ $\mathrm{Hz}$.

$$
\begin{aligned}
\mathrm{NEP}^{2} & =N E P_{p h}{ }^{2}+N E P_{J n}{ }^{2}+N E P_{s q}{ }^{2} \\
N E P_{p h}{ }^{2} & =\gamma 4 k_{B} T^{2} G\left(\frac{1}{1+\omega^{2} \tau^{2}}\right) \\
N E P_{J n}{ }^{2} & =\frac{4 k_{B} T}{R_{T E S}|S|^{2}}\left(\frac{\tau}{\tau_{0}}\right)^{2}\left(\frac{1+\omega^{2} \tau_{0}{ }^{2}}{1+\omega^{2} \tau^{2}}\right) \\
N E P_{s q}{ }^{2} & =i_{n}{ }^{2}(S Q U I D) \cdot|S|^{2}
\end{aligned}
$$

where $\gamma$ is a factor on the order unity, $\mathrm{S}$ is the responsivity of the device, $\tau$ is the effective time constant, and $\tau_{0}=\mathrm{C} / \mathrm{G}$. The terms describe the thermal fluctuation noise, Johnson noise, and amplifier noise respectively. Above $200 \mathrm{~Hz}$ the measured noise current is about $20 \%$ above the expected phonon noise. The small excess could result from systematic errors in estimating the $\mathrm{G}$ and transition temperature. For the lower bias voltage shown in Figure 3, the device shows an optical time constant of $1.1 \mathrm{~ms}$, and gives a figure of merit NEP $\sqrt{ } \tau=6.6 \times 10^{-19} \mathrm{~W} \cdot \mathrm{s}$.

\section{CONCLUSIONS}

With the low excess noise performance, fast time constants, and understood thermal behavior, our device is a promising approach to millimeter wave detection. Currently we are measuring the complex impedance of our TES, which will yield a more thorough characterization of the device. From these measurements, we will obtain detailed information about the heat capacity and loop gain through the superconducting transition. We are beginning tests of the antennacoupled structure and bolometer sensitivity to $140 \mathrm{GHz}$ coupled radiation. 


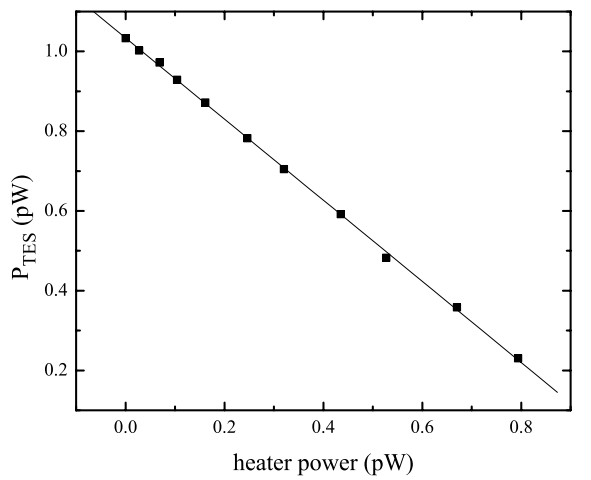

Figure 4: Quiescent power in TES during the superconducting transition vs. applied input heater power. The slope of the line, $-\delta$ Ptes/ $\delta$ Pheater $=$ $1.0 \pm .1$, implying the loop gain for our device is large.

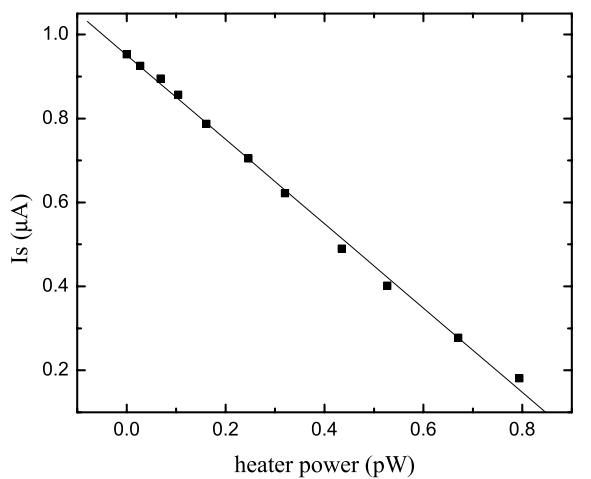

Figure 5: Current response of TES to input heater power, biased at $1 \mu \mathrm{V}$. Our measurements show to within $1 \%$ that the current responsivity of a TES is given by $-1 / \mathrm{V}_{\mathrm{b}}$ with an accuracy of $1 \%$.

\section{REFERENCES}

${ }^{1}$ Lee AT, Richards PL, et al, A superconducting bolometer with strong electrothermal feedback, Appl. Phys. Lett. 69(12), 1801-1803

${ }^{2}$ Vayonakis A, et al, The millimeter-wave properties of superconducting microstrip lines, Low Temperature Detectors, AIP Conference Proceedings from LTD-9 2002

${ }^{3}$ Goldin A, et al, SAMBA: Superconducting Antenna-coupled, Multi-frequency, Bolometric Array, Low Temperature Detectors, AIP Conference Proceedings from LTD-9 2002

${ }^{4}$ Irwin, KD, An application of electrothermal feedback for high resolution cryogenic particle detection, Appl. Phys. Lett. 66 (15), 1998-2000

${ }^{5}$ Lee SF, Gildemeister JM, et al, Voltage-biased superconducting transition-edge bolometer with strong electrothermal feedback operated at 370mK, Appl. Optics, 37 (16), 3391-3397 